مجلة جامعة الملك عبدالعنيز: الاقتصاد الإسالامي، م31 ع3، ص ص: 67-80 (محرم 1440/ أكتوبر 2018)

DOI:10.4197/Islec.31-3.4

\title{
نحو موجة ثانية لإحياء الوقف في العالم الإسلامي
}

\author{
طارق عبد الله \\ أستاذ مشارك، علم الاجتماع، كلية العلوم الإنسانية والاجتماعية

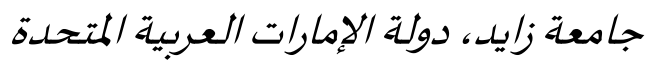

المستخلص. تركز أغلب الأدبيات العبية المعاصرة ذات الصلة بموضوع الوقف على مسألتين رئيستين؛ تتعلق الأولى ببيان مفهومه وحكماه وشروطه. وترتبط الثانية، بالأدوار التي قام بها التها الوقف في مستويات اجتماعية وثقافية واقتصادية مختلفة، مع دعوات وتوصيات لتفعيل الوقف في مجتمعاتنا المعاصرة. غير أن هذه الأدوار، كما جاءت مثلاً في الورقة الرئيسة للدكتور

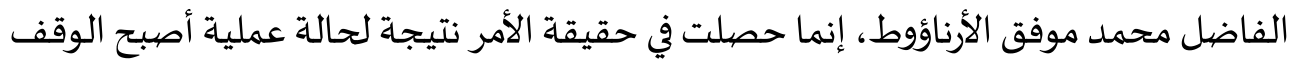

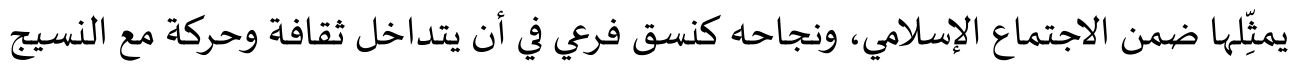

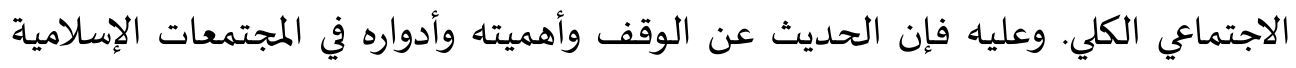
المعاصرة إنما يرتبط في حقيقة الأمر بتوفر شروط قيام هذه الحالة وإمكانية إعادة بنائها بمفردات الحاضر، ورسم مستقبل الوقف ضمن متطلباتها. وهذا ما تتناوله الورقة وتسلط الضوء عليه عبر ست فقرات مشفوعة بخاتمة تتضمن أهم النتائج والتوصيات. الكلمات الدَّالة: الوقف، إحياء الوقف، الأنساق الاجتماعية. تصنيف E21, E22, E23 : KAUJIE 


\section{1- الوقف كحالة اجتماعية: ثلاثية تأسيس النسق}

ولقد استلزم هذا التوجه بناءً مؤسسيًا وفق ضوابط فقهية وقانونية وإدارية، عمل على تهيئة مناخ عملي للارتقاء

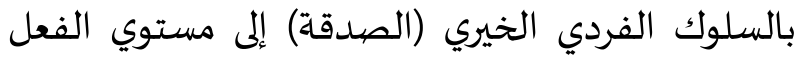
الاجتماعي (الصدقة الجارية)، مما أفرز نسقًا اجتماعيًا

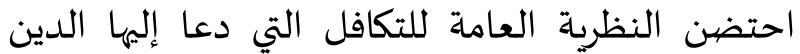

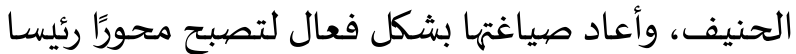

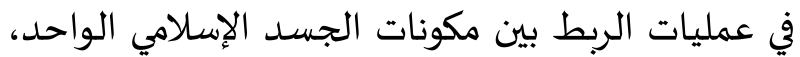

بغض النظر عن حدود مكوناتها الجغرافية(2). من ناحية ثانية،، مثل "المجال العام" الموقع المميز للنشاط الوقفي(3). ولقد ساهمت الخبرة الحضارية المتراكمة للمجتمعات المسلمة في إنشاء آليات مدنية متعددة مكنت شرائح كثيرة من تسخير جزء مهه من

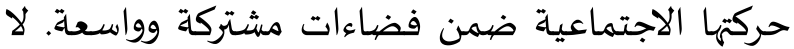
شك أن الوقف قد أصبح خلال فترة امتدت من القرن التاسع حتى نهاية القرن السابع عشر الميلادي الحلقة الرئيسة في هذه الآلية، ووفر من خلال مرونة صيغها إمكانيات واسعة للعديد من القوى الاجتماعية لتساهم في

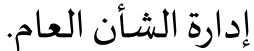
من ناحية ثالثة استطاع القطاع الوقفي أن يصبح "سُلْطة" بالمفهوم الاجتماعي ( Deguilhem and Henia, 14 : 2004)، أو ما يمكن وصفيه بالقوة الناعمة التي تمكنت من أن تثبّتِ موقعها ضهمن السياق العام، من خلال الدور

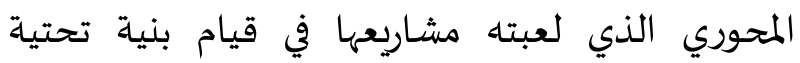
اجتماعية، واقتصادية، وثقافية شكلت مجتمعة أحد ركائز المدينة الإسلامية. في هذا الإطار سمحت المؤسسات

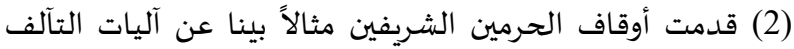
العملية ما بين المسلمين باختلاف مناطقهم الجغرافية، (أنظر: .Hoexter, 1998 Hoexter, 2002: (3) أنظر: مريم هوكستر "الوقف والمجال العام"
يركز التحليل الاجتماعي على رصدد الأنماط السلوكية التي

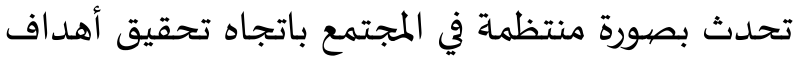

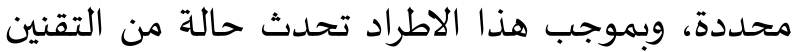

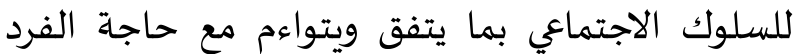
والمجتمع. في هذا السياق، تطرح نظرية الأنساق لهاق

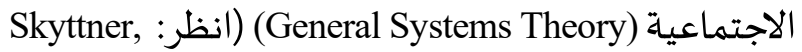

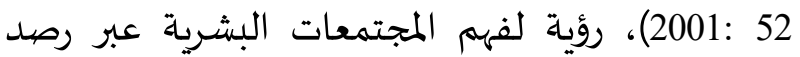

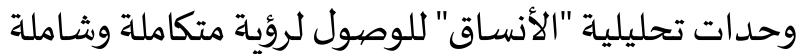
في النظر إلى المجتمع وحركته وبالتالي في فهم (holistic) التفاعل الحاصل بين مجمل عناصره. تتشكل النشاطات

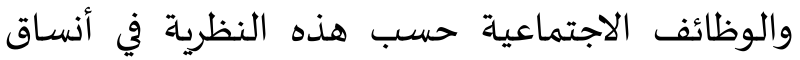
رئيسية وأنساق فرعية تترابط فيما بينها من خلاجل العلاقات التبادلية التي تحدث بين كافة الظواهر والدوافع لـانع

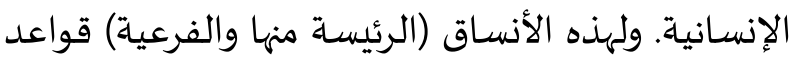
تحكم علاقاتها مع بعضها، مما يؤثر على مخرجاتها

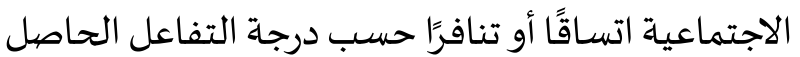

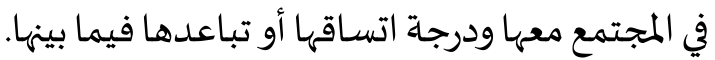
وإذا ما اعتبرنا الوقف نسقًا اجتماعيًا فرعيًا، (أنظر: يمكننا القول بأنه تأسس وفق ثلاثيًا فرعيًا، (Abdallah, 2001: 37

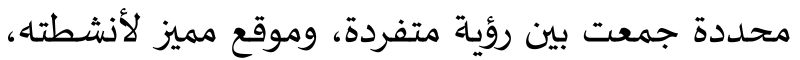
وسلطة اعتبارية ومادية مارس عبرها مهامه الرئيسية. أما الرؤية فهي المعبرة عن جوهر الوقف الذي ربط

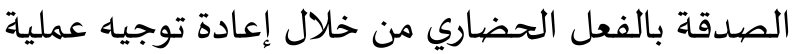

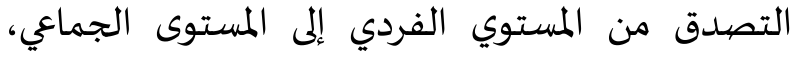
واستطالة أثرها عبر جريان نفعها، بدل آنية حركتها(1).

(1) لا نناقش هنا أهمية الصداقة الفردية التي تمثل أحد أهم ركائز

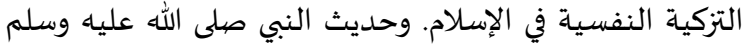

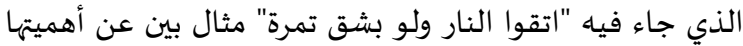

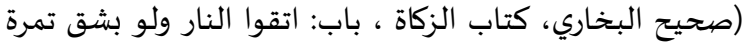

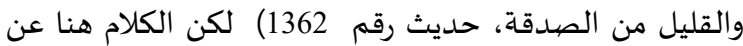

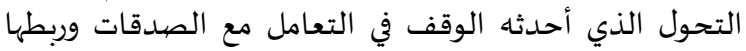

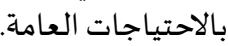


بنية تحتية متكاملة لمؤسسات العلم والمعرفة، (المصيدر السابق، 17-16). فإضافة إلى إنشاء المؤسسات التعليمية أدت حركة الوقف إلى نشأة أحياء سكنية ترتبط بالاهتمام إلىاء الماء بالمهاجرين من طالبي العلم ومن التجار ومن المسافرين الغرباء، وتوفير أماكن لراحتهم وتسهيل مهامهم في البلدان الإسلامية الأخرى. فعلى سبيل المثال نجد "حي المغاربة" نسبة إلى المسافرين القادمين من بلدان الشمال الإفريقي-

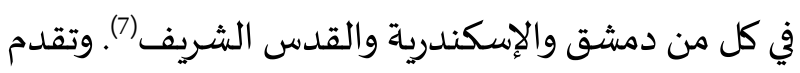
أوقاف الحرمين دلالات عميقة على عمليات التداخل التي تنتجها المشاريع الوقفية بين الجوانب الاقتصادية والثقافية والسياسية والوجدانية ذات العلاقة بتقوية المناعة الذاتية للشعوب المسلمةة، ونقل فلسفة التآزر والتواد بين المسلمين من المستوي الوجداني إلى المستوي العملي. مثلت أوقاف الحرمين على سبيل المثال الترجمة

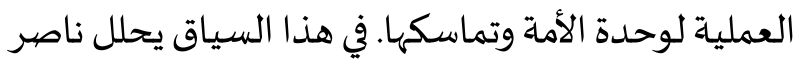
السعيدوني نموذج أوقاف الحرمين في الجزائر العثمانية

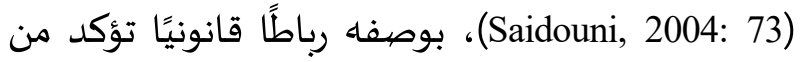
خلاله الجزائر العثمانية انتمائها للأمة الإسلامية. وهي كذلك رمز للأخوة الإسالامية حيث يضيف الكاتب بأن "حصة الحرمين من ريع هذه الأوقاف كانت تمثل بالنسبة لأفراد الشعب [الجزائري]ٍ] مهمة كريمة وواجب نبيل يعكس العلاقات الروحية والثقافية والاقتصادية بين الجزائر العثمانية من ناحية ومكة والمدينة من ناحية ثانية"،

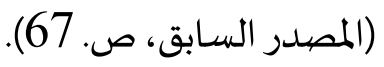

وفق ثلاثية الرؤية، والموقع والسلطة تشكلت الأوقاف كقوة مجتمعية دافعة لعبت جملة من الأدوار، وساعدت على استدامة تماسك المجتمعات الإسلامية رغم الكثير من

(7) The Story of Abu Madein Al-Ghouth's Legacy (Waqf)to the Moors in Jerusalem (https://english.palinfo.com/176.
الوقفية أن تترافق حركة الأفكار والرجال مع حركة

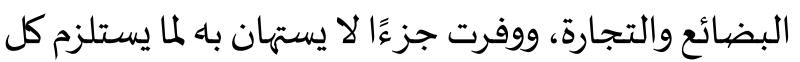
هذه الحركة من وسائل واحتياجات. مثلت الأراضي الزراعية التابعة للأوقاف نسبًا عالية من إجمالي الأراضي الصالحة للزراعة في كثيرٍٍ من الدول

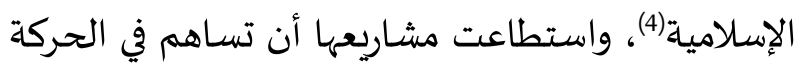
الاقتصادية وتُكوّن قطاع جذب اقتصادي لا يستهان باه.

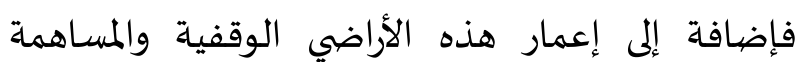
المباشرة في توفير الاحتياجات الزراعية الضرورية وتوفير فرص عمل، نشأ اهتمام متزايد بصناعة وسائل النقل مثل السفن الكبيرة لإيصال المواد الغذائية التي ترسلها مؤسسات الأوقاف إلى كثير من البلدان الإسلامياة، وما

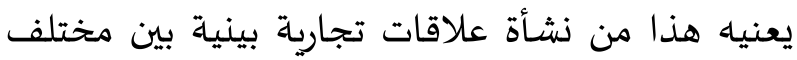
الدول الإسلامية(5) وانعكاس ذلك على الروابط السياسية والاجتماعياة بينها، وتقليل اعتمادها على البضيائع والمعونات الخارجية. ولم يكن غريبا بالتالي أن تساهم الأوقاف في مسائل تتعلق بالسياسات الخارجية للدول الإسلامية، حيث مثلت أحد الأدوات التي استعملتها هذه الدول لإدارة

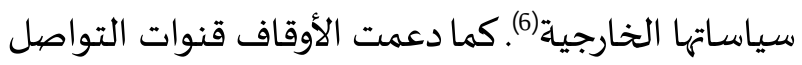
بين العلماء المسلمين، وكانت أحد الروافد الأساسية لتوفير

(4) لعل أبرز الأمثلة لما وصلت إليه هذه النسبة في الدولة العثمانية في

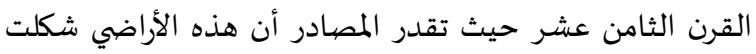

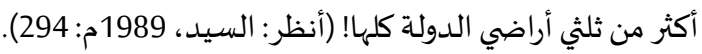
(5) جاء في حجة وقف زوجة السلطان سليمان القانوني التي ترجع إلى الى

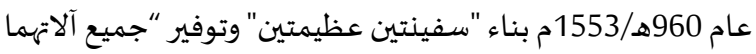

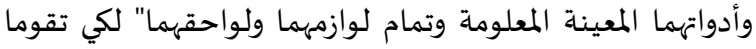

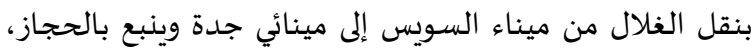

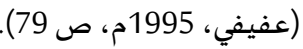

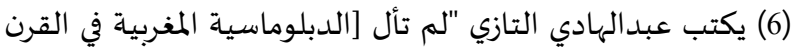

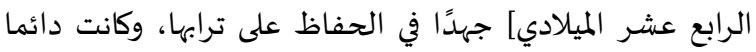

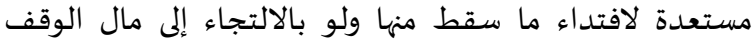

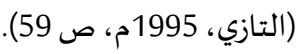


الإطار نماذج إدارية لهيئات حكومية مستقلة تستهدف تطوير القطاع الوقفي وصرف ريعه حسب شروط الواقفين وكذلك الدعوة لأوقاف جديدة برؤية تجمع بين التمسك بأحكامه الشرعية من ناحية، وربطه باحتياجات المجتمع التنموية من ناحية أخرى(9). ما حدث كان "ثورة" فئه بكل المقاييس حيث تعددت الدعوات لإعادة النظر في إمكانيات الوقف التنموية، بل وطرح رؤى متقدمة لنماذج تستفيد من الصيخ الوقفية. لكن بمجرد انقضياء العشرية الأولى (1990-2000م) تراجعت الموجة الأولى، أو على الأقل لم تتطور بالشكل الذي يمكنها من المرور لمراحل نوعية متقدمة. ما يزال العالم الإسلامي ينتظر موجة ثانية تستكمل ما البدايات باه بعد ما يقارب ربع قرن من الزمان حيث إن مقاربة الوقف لا تتم فقط عبر "تحديث" وسائله الإدارية على أهميتها، ولكن المسألة تتعلق في أصلها بإعادة الاعتبار لحركته الكلية على أساس احترام وتفعيل الثلاثية المؤسيّسة لمنظومته، عبر تأكيد رؤيته المتفردة، وتحصين

موقعاه ضمن النسق الكلي، وتثبيت سلطته الاجتماعية. وهذا ما بينته تحديدًا التجربة الغربية المعاصرة في التعامل مع أشكال التبرع والوقف تحديدًا الذي أصبح يمثل دورًا محوريًا في تشكيل النسيج الاجتماعي والاقتصادي الغربي، على أساس تصور متكامل للمجتمع يحدد لنماذج التبرع المختلفة دورًا واقعيًا مباشرًا في الحياة العملية، إضافة إلى توفير مناخ تشريعي وسياسي واقتصادي لا يتعارض وهذا الدور بل ويفتح له إمكانيات

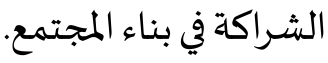

(9) يبقى المثال الأبرز في هذا الصيدد نموذج "الأمانة العامة للأوقاف

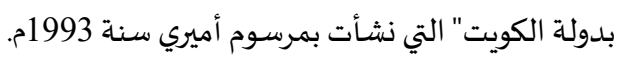

الاختلالات السياسية التي شهدها تاريخها(8)، وأصبحت بذلك أحد العناوين الرئيسة للحضيارة الإسلامية. برز الوقف في علاقتاه بإدارة مسألة الضبط الاجتماعي وبناء شبكة من العلاقات الاجتماعية والاقتصادية والثقافية الكثيفة، التي سمحت ولفترات طويلة من تحقيق ديمومة هذه المجتمعات وتطور لافت لنظمها، وبالتالي دعم ثبات العالم الإسلامي كحضارة عالمياة.

\section{2- الوقف في القرن العشرين: موجة الإحياء الأولى}

شكل إعادة الاهتمام بالأوقاف أحد العلامات الفارقة

التي ميزت آخر عشرية من القرن العشرين في العديد من البلدان الإسلامية، حيث تنامت الدعوة للوقف وتزايد منسوب الاهتمام بصيغاه، مع تطور لافت لأنشطة علمياة اتخذت من الوقف موضوعها الرئيسي. ويمكن القول بأن ما جرى خلال هذه العشرية مثّل "موجة أولى" قطعت مع

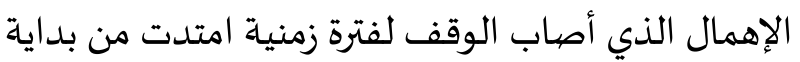
الحقبة الاستعمارية وحتى تسعينات القرن العشرين، ولهذا السبب تحديدًا استهدفت هذه "الموجة" إعادة إحياء سنة الوقف ومحاولة نشر ثقافته سواء من خلال التعريف بفقها وأحكامه، أو من حيث إبراز نماذجها مأه التاريخية والنظر في الأدوار التي قام بها داخل المجتمعات الإسلامية. في هذا السياق ظهرت بعض المحاولات لإعادة تطوير أداء المؤسسات الوقفية الرسمية (الوزارات) عبر إعطائها مرونة أكبر لإدارة الأنشطة الوقفية، وبرزت في هذا

(8) سمح نظام الخلافة كنظام سياسي فضفاض بهامش من الحركة

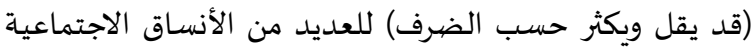

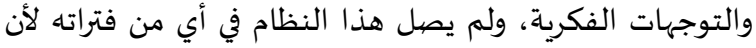

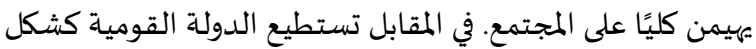

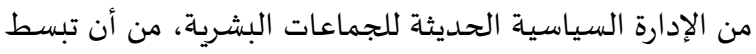

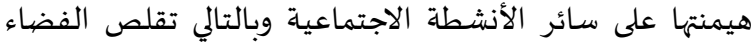

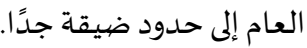


مؤسسة القضاء جهاة مرجعية للحسم في قضايا النظارة على الأوقاف بتوفر شروط أساسية، لعل من أهمها

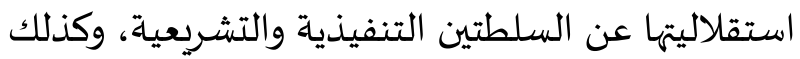
كفاءتها في الرقابة وحسم المشاكل أي تخصصيها في المسائل

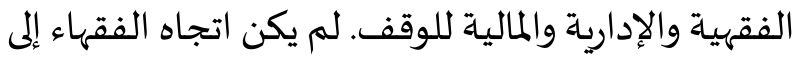

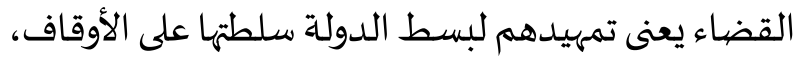
وإنما عملوا على الاستفادة من مؤسسات الدولة الرقابية والإدارية لتحصين الوقف وتحقيق شروط الواقفين. ويتضح هذا المنتى جليًا فيما ذكره جلال الدين السيوطي من موقف القاضي توبة بن نمر بن حومل الحضريكي

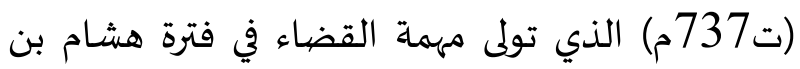

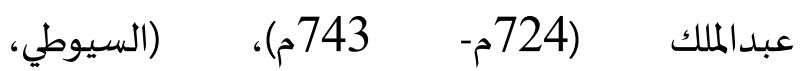

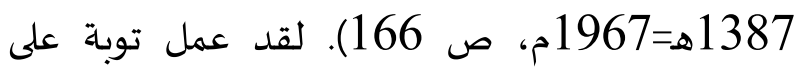
الاستفادة من النهضة الاجتماعية والاقتصادية التي ميزت

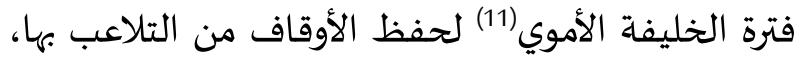

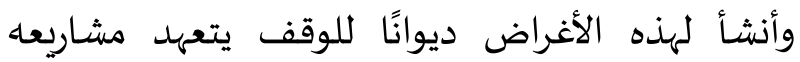
ومؤسساته بالرقابة ومحاسبة تقصير النظار وتهاونهم في

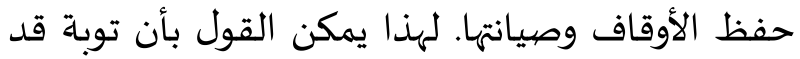

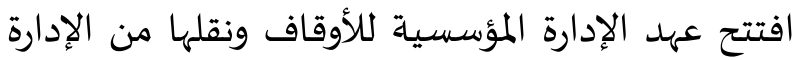

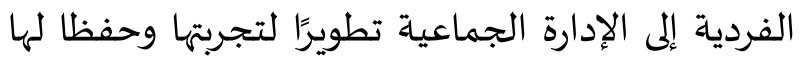
ضيد التلاعب.

على هذه الأسس بنت المؤسسات الوقفية نفسها

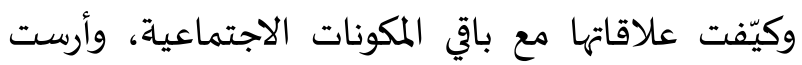
تقاليد عملية للتعاون مع القطاعات الاجتماعية وفي باتي مقدمتها الدولة. لقد استندت علاقة الوقف مع هذه

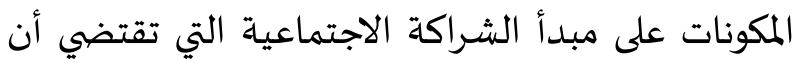

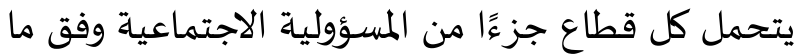
يتميز باه من سلطات (اقتصادية كانت، أم سياسية أم

(11) اهتم هشام بن عبدالملك (724م-743م) خلال فترة حكمها بتنظيم الدواوين، وبالإصلاح الزراعي، ورعاية المبات العلم والثقافة، وترجمت في عهده الكثير من المؤلفات.

\section{3- الوقف والدولة: دروس التاريخ}

لقد تطورت مؤسسات الوقف ضمن التحولات الاقتصادية والاجتماعية والعلمية للحضيارة الإسلامية حيث حتمت البيئة البسيطة التي ميزت الدولة السلطانية في بداياتها تواضع الإدارة الوقفية، فكانت فردية بامتياز.

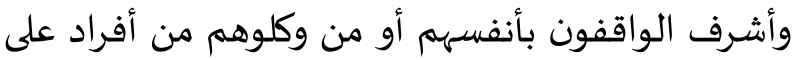
متابعة صدقاتهم الجارية وتحقيق مقاصدهمه. ثم تطورت

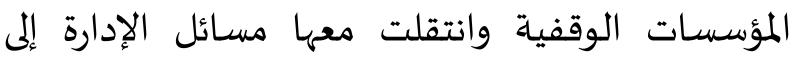

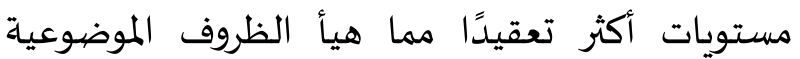

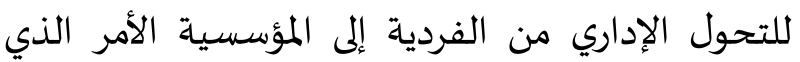

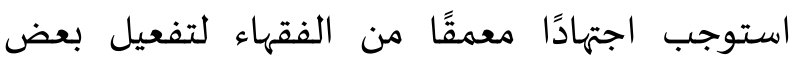

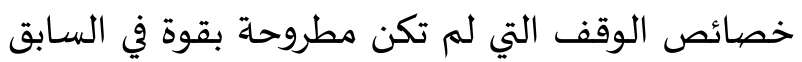

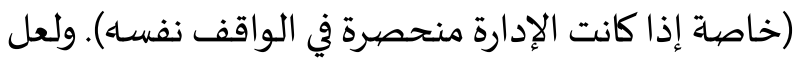
أهم هذه الخصائص التي استدعت نقاش الفقهاء ما

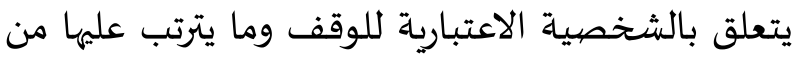

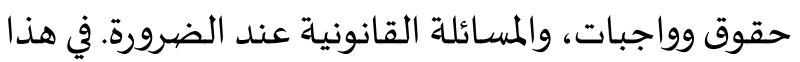

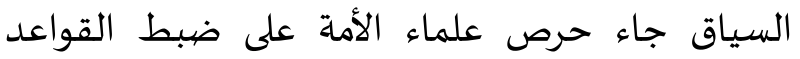

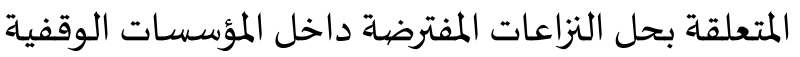
والتصدي لكل إمكانيات الفساد الإداري والأخلاقي. ولهذا

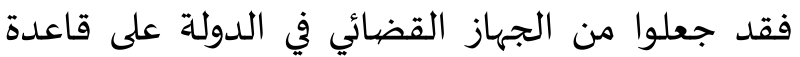

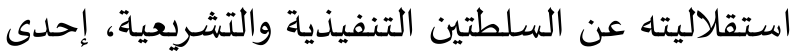

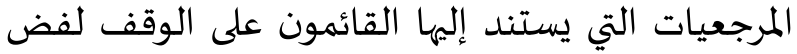
الخلافات التي يكون الوقف أحد أطرافها.

لقد حمّل الفقهاء القضياء دورًا أساسيًا في مراقبة وتقييم النظارة على الوقف على خلفية تطور مؤسسة القضاء ذاتها، واختصاصها في حسم النزاعات، ونزاهتها

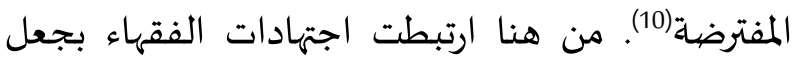

(10) ارتبطت اجتهادات الفقهاء بجعل مؤسسة القضياء جهة مرجعية

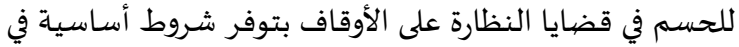

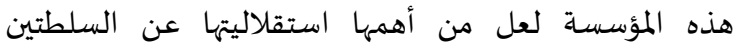

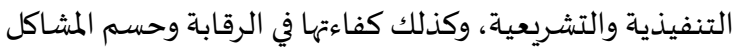

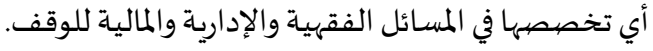


المؤسسات الوقفية وإلحاق ما تبقى منها بإدارات حكومياة،

$$
\text { وسن تشريعات تقنن هذا التوجها. }
$$

تضافرت العوامل السابقة لتنتهي في الأخير إلى وصاية

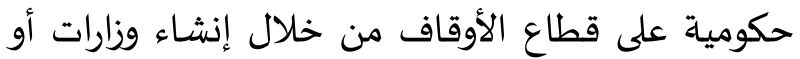
هيئات تقع على عاتقها إدارة الأوقاف بشكل تام، وتنفيذ ما

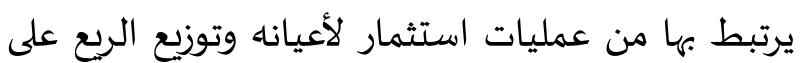

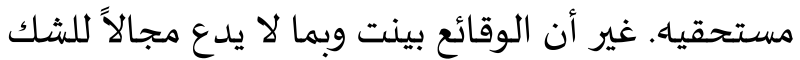

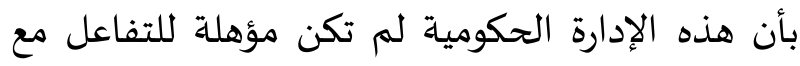
ديناميكية الوقف الاجتماعية وللاستفادة من قدراتها. لقد خسر الوقف في هذه العملية كثير من مميزاته وفي وقت كانت مؤسساته بحاجة ماسـة إلى الكثير من الإصلاح لا

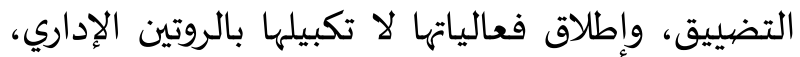
والاستفادة من قدراتها لا إهمالها. في هذا السياق وقع التضيليق الشديد على حرية الحركة التي تتمتع بها المؤسسة الوقفية من خلال إدماجها ضمن نسق إداري مركزي يعتمد بالدرجة الأولى على الوسائل البيروقراطية في مسائل الصرف والاستثمار. ومن ناحية ثانية ترافقت إدارة الأوقاف من المؤسسات الحكومية بعدم احترام شروط الواقفين بشكل تام، حيث أسست التشريعات القانونياة، الخاصة بإعادة ترتيب أوضاع الأوقاف داخل الإدارة

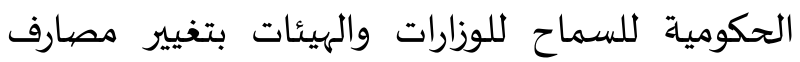
الوقف دون الرجوع إلى الواقف أو دون التقيد بشروطه (15).

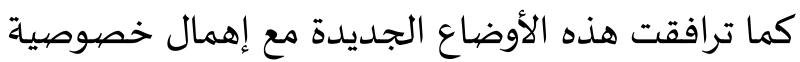

(15) يحلل المستشار طارق البشري تطور القوانين المصرية في هذا الاتجاه

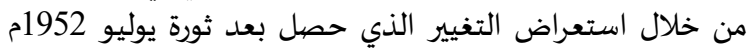

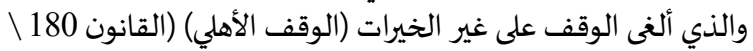

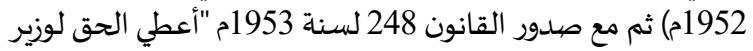

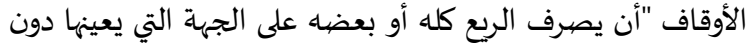

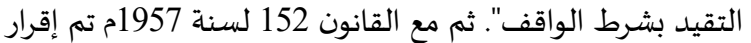

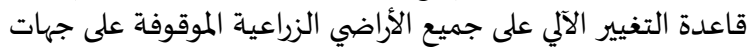

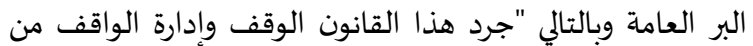
الأعيان ذاتها وقرر الاستيلاء على الأراضي الزراعية الموقوفية المادية" (أنظر: البشري، 2003م. ص ص: 674-675).
اجتماعية). وما يجب التركيز عليه في هذا الباب هو أن

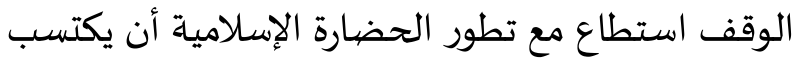
جزءًا مهمًا من السلطة الاجتماعية والاقتصادية في المجتمعات الإسلامية ويجيرها لصالح الأمة، في مقابل سلطة الدولة المرتبطة بمصالح الطبقة السياسيةي(12)، أو الو سلطة القطاع الخاص ذات العلاقة بالمنفعة المادية. 4- مقاربة الدولة الحديثة للوقف إن تحمل المؤسسات الرسمية مسؤولية إدارة الأوقاف في أغلب دول العالم الإسلامي ما بعد فترة الاستعمار

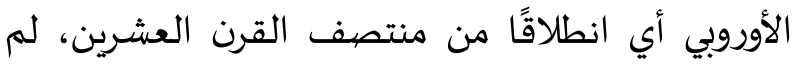

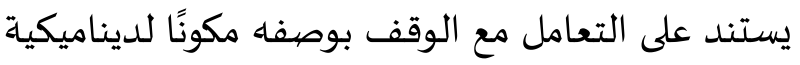
اجتماعية متوازنة، وإنما تأسس على تصيور للدولة الحديثة

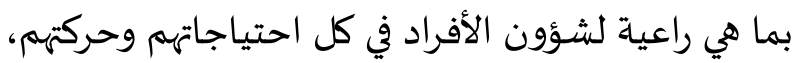

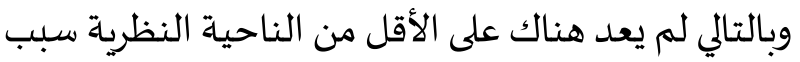

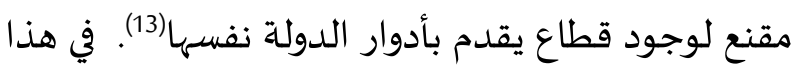

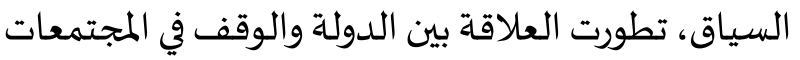

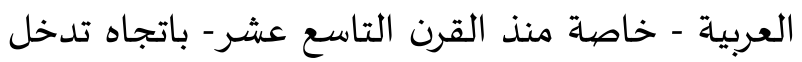
تدربجي في شؤونها ومحاولات متعددة لتقييد حركتاه وقد مداهد وصلت في العديد من الحالات إلى تقطيع أوصاله وإلغاء أجزاء منه(14) والتجرؤ على اغتصاب أعيانها، وانهات وانتهت في منتصف القرن العشرين إلى إحداث ولاية كاملة على إلى

(12) لعل التخوف من استعمال هذه المصالح السياسية في غير موضيعها،

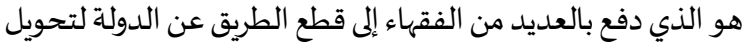

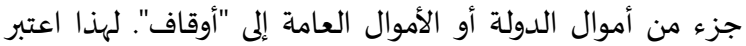

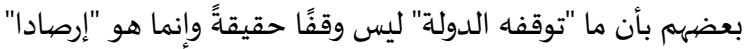

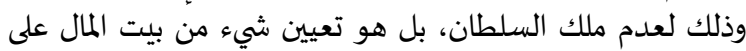

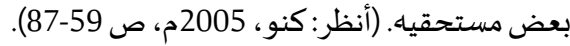

(13) Pierre Rosanvallon, La Crise de l'Etat-providence, Editions Seuil, France 1976.

(14) تم إلفاء الأوقاف الأهلية في العديد من البلدان العربية مثل سوريا

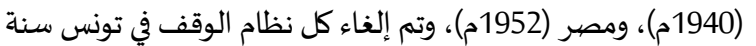

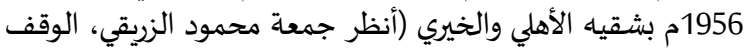

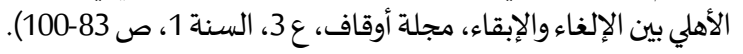


ضروري للإججابة عن التساؤل التالي: ماذا يريد المسلمون من الوقف في الوقت الحاضر؟

أولاً: يتصددر مفهوم الدولة الراعية إعادة النظر هذه، لأنه "انتهى" نظريًا وعمليًا منذ سبعينات القرن العشرين (Pierre Rosanvallon, op. cit) السياسي للبلدان الغربية حيث تجاوزت هذه البلدان عقدة "الدولة الراعية" وأسست للدولة التشاركية التي تتعاون فيها قطاعات المجتمع الثلاثة المعبرة عن القوى الاجتماعياة

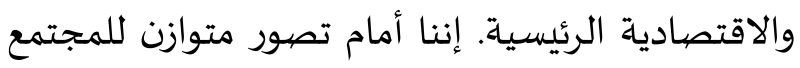

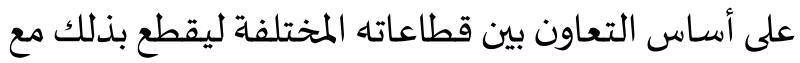
الدولة المتغولة، والقطاع الخاص المتوحش، والقطاع الثالث المنفلت من كل ضوابط في الوقت نفسـا تقر هذه المهاه الرؤية التشاركية بأهمية الدولة وأجهزها التنفيذية

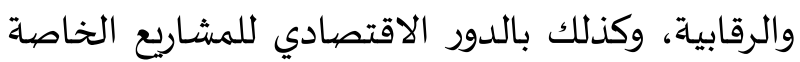
ومشروعية السعي للرتح طالما كان هذا وفق القانون

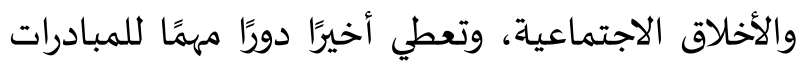

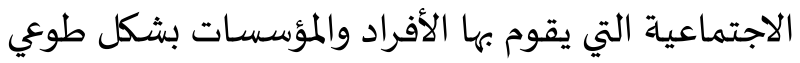

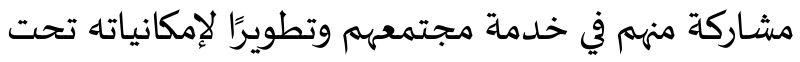
مظلة القانون وعين المؤسسات الرقابية. ثانيًا: إن تأكيد علاقة الوقف بالمجتمع المدني مسألة جوهرية لأهنا تعكس فلسفتا بما هي مساهمة الأفراد بشكل طوعي في تلبية احتياجات اجتماعية، ولأن هذا الانتماء يبين مسألتين مترابطتين.

فمن ناحية تظهر عبقرية الوقف في إعطائه الصدقة

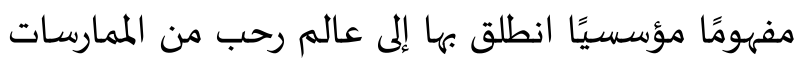

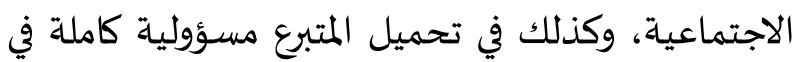
التخطيط الدقيق لهذا البناء المؤسسي ليصبح الواقف شريكًا مباشرًا في صنع مستقبل المجموعـة.

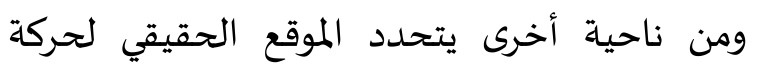
الإيقاف ضمن شراكة مبدعة بين القطاعات المختلفة:
المال الوقفي بدمجه في بعض الحالات مع الأموال الحكومياة، وبعدم التخصص في استثماره وتنميته. لقد بات من المؤكد بأن بسط الدولة سلطتها الإدارية والتشريعية على الوقف انتهت بخسارتين فادحتين: تمثلت الأولى في إضعاف أكبر لنظام الوقف من خلال الإجراءات

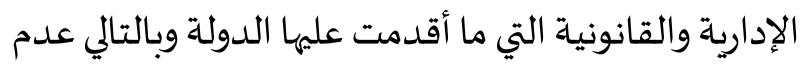
التصدي لما اعترى الوقف من وهن، بل وتعميقها.

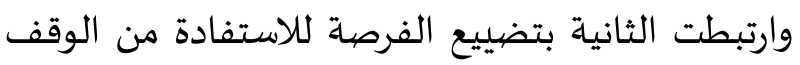
كنسق اجتماعي يساهم في توازن المجتمع وتنميتا بشكل

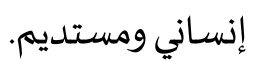

\section{5- ماذا يريد المسلمون من الوقف في الوقت الحاضر؟} يمثل التعامل مع الوقف أحد المؤشرات ذات الدلالات

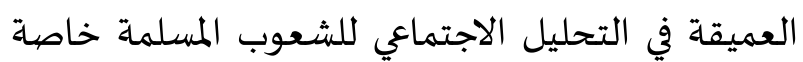
فيما يتعلق بالبحث عن أفضل السبل لبلوغ التوازن والسلم الاجتماعيين. وحيث لا يمكن الفصل بين حركة الأفراد واتجاهات سلوكياتهم داخل مجتمعاتهم من ناحياة،

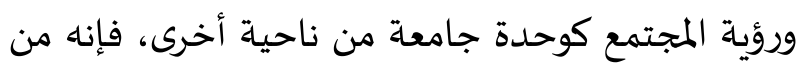
الصعب اختزال الوقف في أوعية مالية (تبرعات) تحتاج إلى

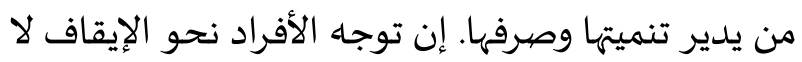
يرتبط فقط بتوفير احتياجات الموقوف علههم بل يتصل كذلك باحتياجات متعددة للواقف ذاته الذي يشارك في صنع بيئة كثيفة من العلاقات يلتقي فيها المجتمع والدين والاقتصاد والوعي الإنساني. في هذا الإطار تصبح عملية الإيقاف أحد البوابات المهمة التي يلج من خلالها الأفراد للفعل الاجتماعي العقلاني، ومساحة واسعة لممارسة جملة من النشاطات ذات الطبيعة المدنية السلمية. إن إعادة النظر في المسلمات النظرية المرتبطة بالنماذج الاجتماعية والتنموية السائدة في العالم الإسلامي مدخل 
الدولة بوصفها مسؤولة عن تنظيم العلاقات بين المواطنين، وحماية حقوقهم وتحقيق أمنهم، والتوجيه

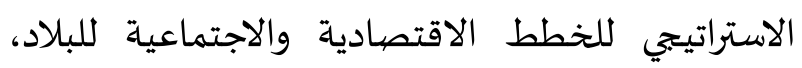

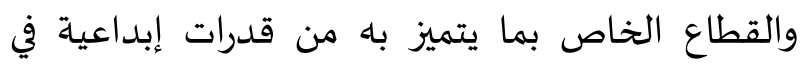

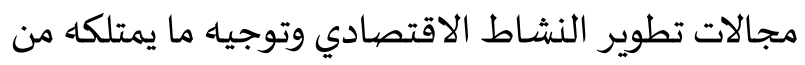

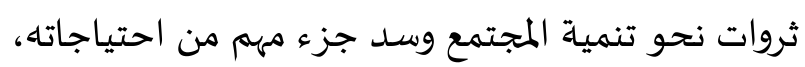
والمجال العام ومن ضمنه مؤسسات الوقف حيث يتمكن

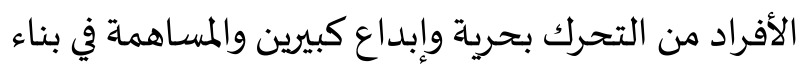
فضاءات مشتركة وواسعة للفعل الاجتماعي.

إن ما يجري على الساحة الوقفية اليوم -مع استثناءات

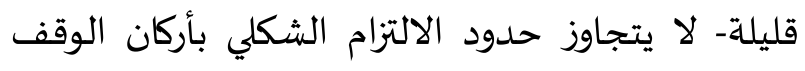
وشروطه الفقهية مع تغييب لجوهره وموقعاه، وسلطته.

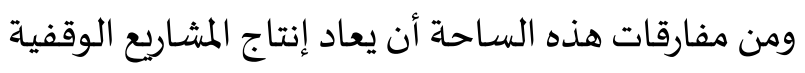

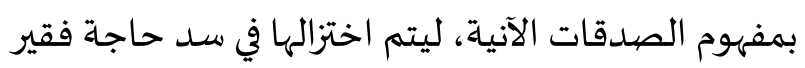

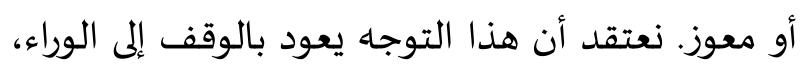

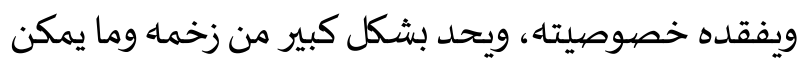

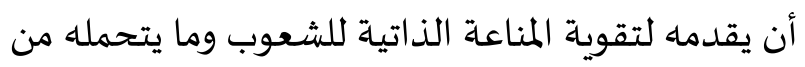
مسؤولية مجتمعية. في هذا السياق يبدو أنه من المهم جدًا، التفريق بين

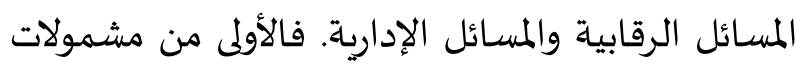

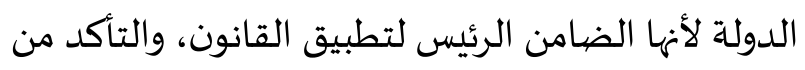

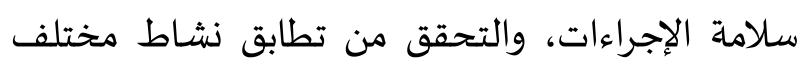

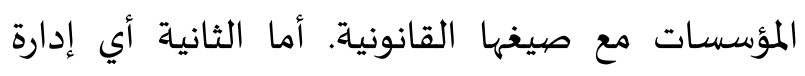

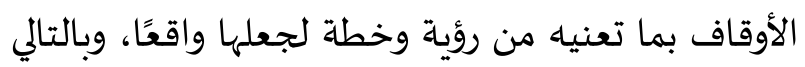

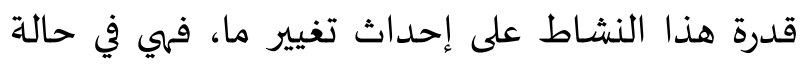

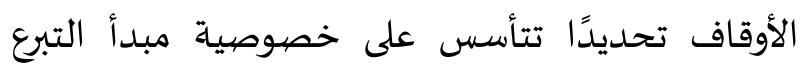

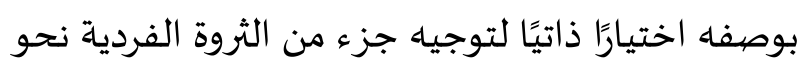

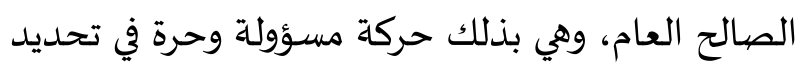

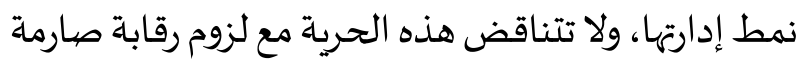

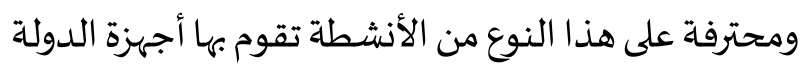

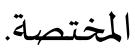


التوجه وتيسر وجود كوادر وقفية تجمع بين التأصيل الشرعي والفكري للوقف من ناحية، والاختصاصات الدقيقة في مجالات الاستثمار والإدارة والتسويق من ناحية أخرى.

\section{6- ماذا يعني أن يكون الوقف آلية تنمية؟}

نعتقد أنه وبعد ثلاثة عقود تقريبًا من عودة الوقف إلى ساحة الفعل الاجتماعي في الكثير من البلدان الإسلامية، قد حان الوقت للمرور إلى مستويات أكثر تطورًا وعمقًا، وأن يتجاوز فهم الوقف والتعامل معه فكرة توفير وعاء مالي يسخر لخدمة حاجة أفراد أو شرائح. آن الأوان لنوجه أنظار المهتمين بالوقف، وكذلك صناع القرار والاقتصاديين والعاملين في مؤسسات المجتمع المدني، إلى الوقف بوصفيه آلية تنموية بامتياز تنطلق من إمكانيات الداخل وتستند إلى الى الموسي حس الأفراد بمسؤوليتهم الاجتماعية، لتطور وتثري المجموعة في مجالات فعلها المتعددة.

تشترك أغلب تعريفات التنمية البشرية على اعتبارها وسيلة للوصول إلى الاستفادة الأصيلة من الإمكانيات المتاحة لتحقيق حياة كريمة وتلبية احتياجات مادية ومعنوية لأفراد المجموعة، دون المساس بقدرة الأجيال القادمة. هذا التعربف هو في الحقيقة وثيق الصلة بحقيقة لاصناد الوقف بوصفه جهدًا مجتمعيًا يعمل على تحقيق كرامة الإنسان في أبعاده المختلفة مع سريان النفع على الأجيال القادمة من الموقوف عليهم. ونعتقد أن هذه العلاقة المباشرة بين مفهوم التنمية البشرية والوقف تمكن المسلمين من إعادة طرح النقد الذي يوجها إلى الاستراتيجيات التنموية التي تمت تجربتها في أغلب بلداننا التي انتهت

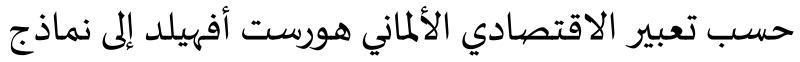

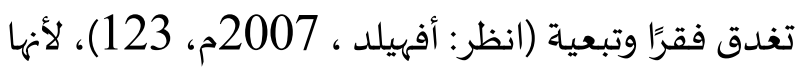
بنيت على نظريات مبتسرة تركز على الأرقام والكميات، وتعتمد في نشأتها وتطورها على العوامل الخارجية.
ثالثًا: لا يمكن للوقف ومؤسساته أن يحدث اختراقًا نوعيا دون جهد علمي أكاديمي متطور (16). ولا يجب أن ننسى الندي أن الحركية التي أحدثها اتساع وتطور نظام الوقف خلال

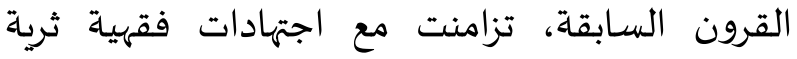
استهدفت الاستفادة المثلي من الإمكانيات التي تمثلها الأوقاف. ومن المفيد هنا التذكير بأن الوقف الذي ساهم بشكل كبير في تمويل العلم طوال فترة التاريخ الإسلامي، ساهم كذلك في تمويل البحث في موضوعه (أي الوقف) حيث تصدت أقلام العلماء لبحث إشكالاته وتطوير تجربته حتى أن القرن التاسع عشر شهد إصدار مجلات مختصة بشكل حصري في موضوع الوقف(17). على هذا الأساس ساهم العلماء في نقاش النوازل المستجدة التي تعترض التجربة الوقفية والبحث عن حلول لها، والعمل على تحسين كفاءة مؤسساتها وتمكينها من ممارسة أدوارها. في هذا لاتجاه يكتب عبدالهادي التازي "لقد اتسعت آفاق تفكير الفقهاء، وأصبحنا أمام ثروة فقهية متنوعة الجوانب التبادي التهاي

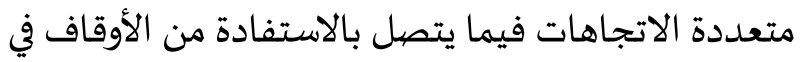
الأغراض التي تحمي الأمة وتضمن سلامتها وتحفظ

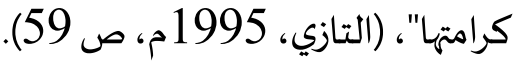

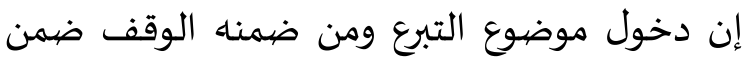
الأجندات الأكاديمية مسألة حيوية لتطوير أدائه. وهذا العمل يلتزم كذلك سلوك منهج منضبط وفق قواعد البحث العلمي الصارمة، ضمن برامج جامعية تدعم هذا

(16) ترعى الجامعات الوقفية الأمريكية برامج جامعية لمرحلتي الماجستير

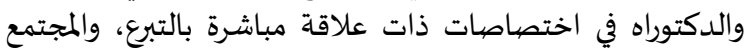

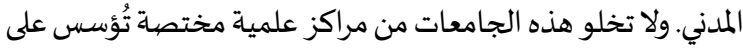

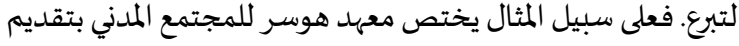

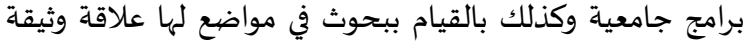

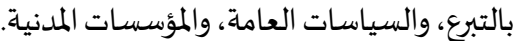
(17) على سبيل المثال صدرت "مجلة الأحباس التونسية" في 1878مات في في التمانية

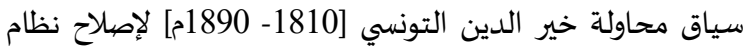
الوقف في تونس، (أنظر : الشيباني بن بلغيث ، أبحاث في تاريخ تونس الإنس

الحديث والمعاصر، مكتبة علاء الدين، تونس، 2008م ، الثبن ، ص285). 
الاستدامة وعدالة التوزيع والتوازن ما بين السعادة المادية

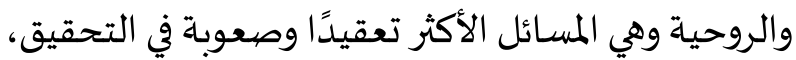
والأقل حضوا في النماذج الاقتصادية بحسب شهرئ شهادات المختصين.

ونعتقد أن توجياه القطاع الوقفي نحو هذا المنحى هي الضرورة التي تحدثنا عنها سابقًا التي تقضي بأن يدخل

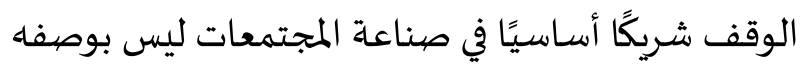
"وعاءً ماليًا" فقط بل من خلال علاقات موضيوعية مع مختلف القطاعات تسمح بتغذية شرايين المجتمع بما يحمله الوقف من قيم وموازنات بين الجوانب المادية

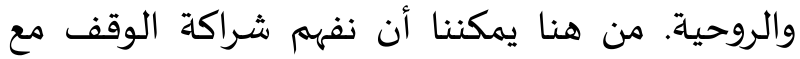
الجهات الحكومية حينما تصبح صيغها جزءا رئيسا من المقاربة التي تجريها الأجهزة الرسمية للعديد من المشاريع الاقتصادية والتنموية عمومًا لبناء القدرات الذاتية للمجتمع والانطلاق من داخله والتعويل على إمكانياته وتشجيع سلوك العطاء وسن القوانين الداعمة له. أما الشراكة مع القطاع الخاص وبالتحديد مع البنوك الإسلامية وشركات التامين التكافلي، فإنها ستسفر عن الاستفادة من صيغ الوقف المرنة لتتجاوز العديد من العقبات والشبهات التي تواجهها حاليًا هذه المؤسسات كالشبهات البروية، وبقائها ضمن فلسفة الربح المتوحش، وتغييبها لجوانب مهمة من مسؤوليتها الاجتماعية. إننا
في المقابل، يبرز نظام الوقف الأكثر حضورًا وتأثيرًا في مجالات تحقيق مقصد العدالة الاجتماعياة، ونقل مفاهيم الأخوة والتآزر والتكاتف إلى سلوكيات وأنشطة عملية، ودفع الحركية الداخلية للمجتمعات الإسلامية، وتطوير قدرات الجماعاة من خلال تحمل الأفراد مسؤولية اجتماعية. كل هذه المنجزات ارتبطت في حقيقة الأمر بفكرة رئيسية مفادها أن الإنسان مسخر لأخياه الإنسان، لكنها لم تنحصر مع الوقف في حدود السلوك الفردي بل تشابكت في ثنايا نظام متكامل له من المقومات القانونية والشرعية

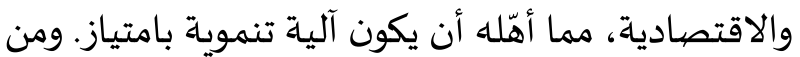
الضروري اليوم أن نضع هذه الآلية ضمن ما يمكن تسميته "بالموجة الثانية للوقف" باعتبار أن الموجة الأولى

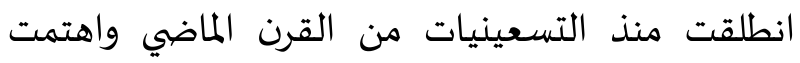
بإعادتاه والتبشير باه وطرحاه ضمن خارطة الاهتمامات الرسمية والأهلية للبلدان الإسلامية. وعليه فإن الموجة الثانية ترتبط بضرورة المرور بالوقف من التركيز على المفهوم إلى الاهتمام بالآلية.

في هذا الإطار نحن بحاجة إلى توسيع ثقافة الوقف وإشاعة القيم الرئيسية التي يتحرك نظاماء من خلالها، وفي مقدمتها تأكيد روح المسؤولية الاجتماعية لكل الفاعلين،

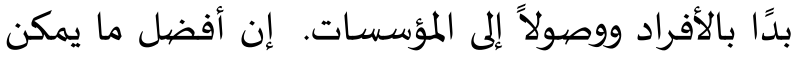

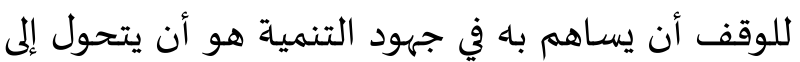

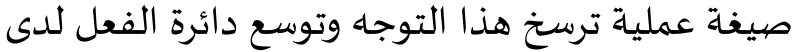
أفراد المجتمع ودفعهم إلى رفض "التواكل الاقتصادي"

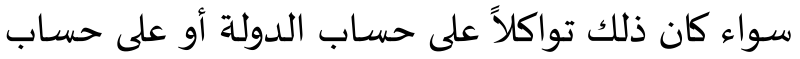
"المحسنين"، وبالتالي تحويل إمكانيات الموقفين إلى "فرص دواء داء

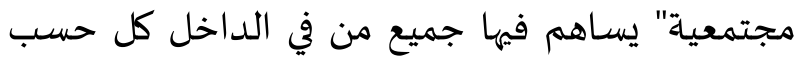
منطقة فعله وما يمتلكاه من ثروة بشرية أو مادية قليلة

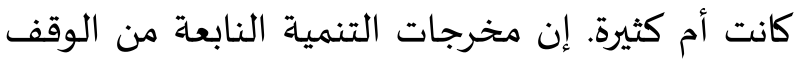
بوصفه آلية من الداخل هي التي تتوافر فيها شروط 
إن الإجابة عن سؤال: ماذا نريد كمسلمين من الوقف؟ تحتاج في هذه المرحلة القطع من تصور مبتسر للوقف وإعادة النظر لمؤسساته بوصفها "كائنًا اجتماعيًا" يحتاج بيئة صالحة للعيش والتطور. و يعتقد الباحث في هذا الإطار أن رسم الاستراتيجيات والعمل على التنسيق ما بين المؤسسات الوقفية في العالم الإسلامي خطوة أساسية لتطوير نوعي للعمل الوقفي بغية زيادة فعاليته الإدارية

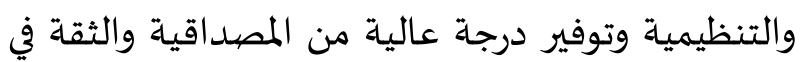
خططه وبرامج عمله لدى الشرائح المتعاملة معه من موقفين ومستفيدين، وبالتالي المرور من مرحلة العمل الوقفي المتناثر هنا وهناك، إلى العمل الوقفي المتكامل ذي الأبعاد والمضامين الحضارية.
نعتقد أن هذه الصيخ ستسمح بتطوير نماذج للبنوك ومؤسسات التأمين أكثر ملائمة لاحتياجات الناس، في لفي الوقت نفساء الذي تحافظ فياه على شرعية معاملاتها دون المساس بنشاطها الاقتصادي. أما الشراكة مع المجتمع

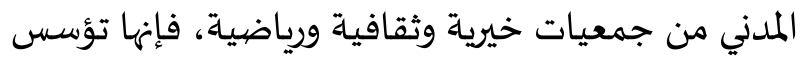
لبناء استقلاليتها وتحميلها المسؤولية في استقطاب موارد وإدارتها والتخطيط لتطويرها الذاتي دون انتظار الإعانات أو البقاء عالة على المنح التي تقدمها الحكومات أو الجهات

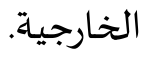
- 7 خاتمة 
البشري، طارق، (2003م). تحولات علاقة الوقف بمؤسسات المجتمع المدني في بلدان وادي النيل. في ندوة: نظام الوقف والمجتمع المدني في الوطن العربي، تحرير: غانم، إبراهيم البيومي، ط1، بيروت: مركز دراسات

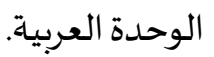

عفيفي، محمد. (1995م). الأوقاف والملاحة البحرية في البحر الأحمر في العصر العثماني في الوقف في العالم الإسلامي أداة سلطة اجتماعية وسياسية، تقديم راندي ديغيليم، الطبعة الأولى، دمشق: المعهد الفرنسي للدراسات العربية. كنو، كينث. (2005م). الإيديولوجيا والخطاب الفقهي. ترجمة أبوبكر أحمد باقادر، مجلة أوقاف، السنة 5، ربيع الإيل

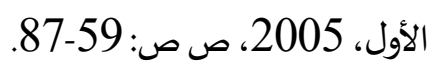
أفهيلد، هورست. (2007م). اقتصاد يغدق فقرًا. ترجمة عدنان عباس علي، سلسلة عالم المعرفة، العدد 335، الطبعة الأولى، الكويت: المجلس الوطني للثقافة والفنون

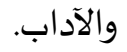

الشيباني بن بلغيث (2008م) ، أبحاث في تاريخ تونس الحديث والمعاصر، الطبعة الأولى، مكتبة علاء الدين، تونس.

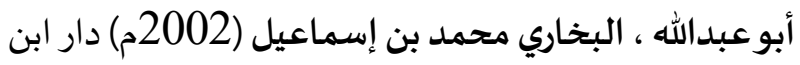
كثير ، الطبعة الأولى، دمشق- بيروت.

Deguilhem, Randi and Henia, Abdelhamid (coordonnateurs). (2004). Les fondations pieuses (waqf) en Méditerranée; enjeux de société, enjeux de pouvoir. Kuwait : la Fondation Publique des Awqaf.

Hoexter, Miriam. (1998). Endowments, Rulers and Community: Waqf al-haramayn in Ottoman Algiers. Netherlands: Brill.

Hoexter, Miriam. (2002). The waqf and the Public Sphere, in M Hoexter, S.N. Eisenstadt and N. Levtzion (editor), The Public Sphere in Muslim Societies, Albany: SUNY Press, pp. 119-138.

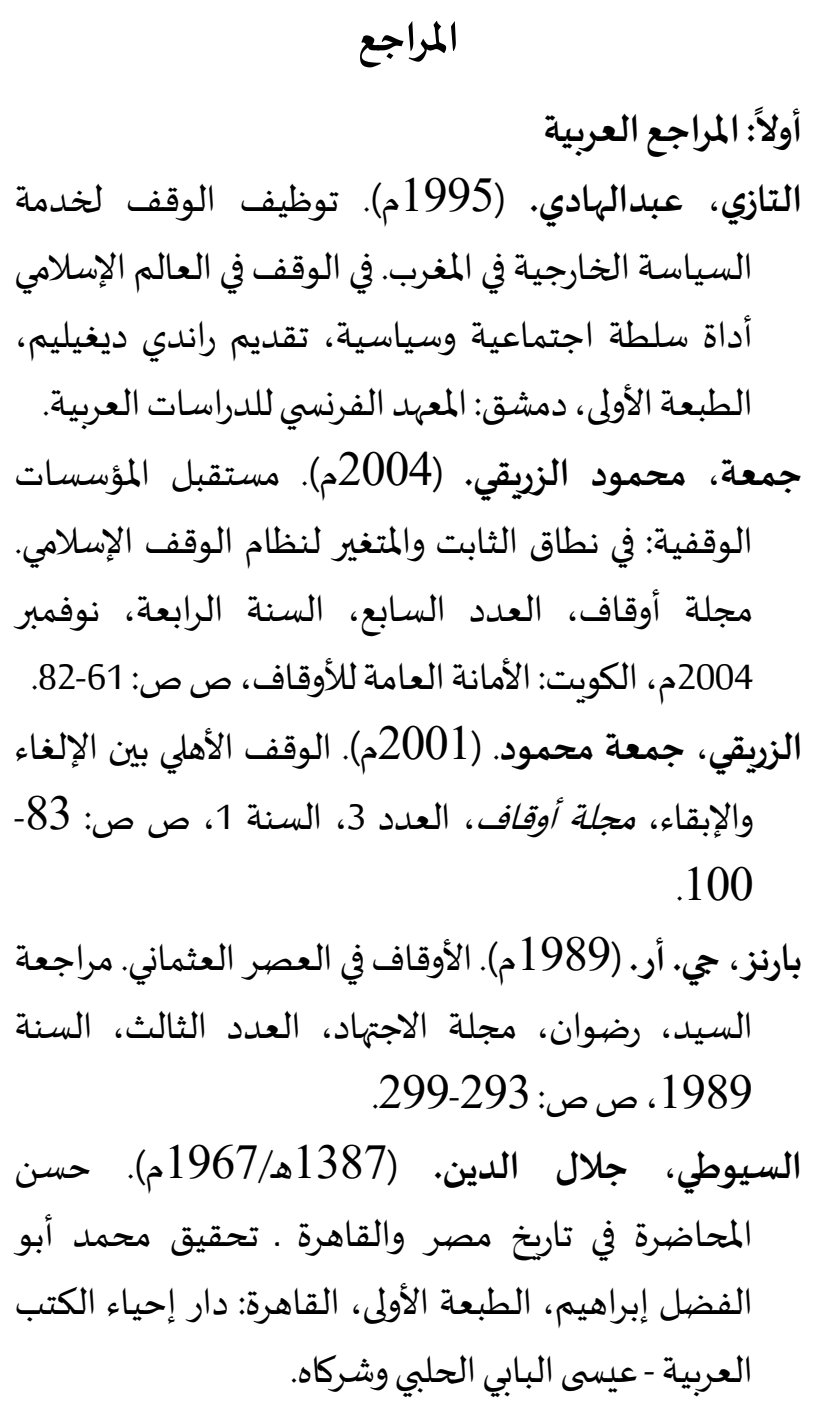

Rosanvallon, Pierre. (1976). La crise de l'Etat Providence. Paris : Editions du Seuil.

Saidouni, Nacereddine. (2004). Les liens de l'Algérie ottomane avec les lieux saints de l'Islam à travers le rôle de la fondation du waqf des Haramayn. AWQAF, $\mathrm{N}^{0} 6$ - Year 3 - June 2004, pp. 37-75.

Skyttner, Lars. (2001). General Systems Theory. London: World Scientific Publication.

Tarak, Abdallah. (2001). Pour une sociologie des awqaf. $A W Q A F, \mathrm{~N}^{0} 1,2001$, Kuwait : la Fondation Publique des Awqaf, pp. 135-165. 
نحو موجاة ثانية لإحياء الوقف في العالم الإسلامي 


\section{Translation of Arabic References}

Afheldt, Horst. (2007). An economy that feeds poverty. Translation Adnan Abbas Ali, Alam Al-Marifa Series, Issue 335, First Edition, Kuwait: National Council for Culture and Arts.

Afifi, Mohamed. (1995). Endowments and Maritime Navigation in the Red Sea During the Ottoman Period. The Endowment in the Islamic World as a Tool of Social and Political Power, Randy Deguilhem (presented), First Edition, Damascus: The French Institute for Arab Studies.

Al-Bishri, Tariq (2003). Changes in the Waqf relationship with civil society institutions in the Nile Valley countries. In, Symposium: The Waqf System and Civil Society in the Arab World, edited by: Ghanim, Ibrahim al-Bayoumi. First Edition, Beirut: Center for Arab Unity Studies.

Al-Suyuti, Jalaluddin. (1387AH/1967 AD). Good lecture in the history of Egypt and Cairo. Mohammed Abu Fadl Ibrahim (critical edition), first edition, Cairo: House of revival of Arabic books - Issa Albabi Halabi and Partners.
Al-Zuriqi, Jumaa Mahmoud. (2001). National Waqf Between Abolition and Retention, Awqaf journal, Issue 3, Year 1, Kuwait: Kuwait Awqaf Public Foundation, pp. 83-100.

Al-Zuriqi, Jumaa Mahmoud. (2004). The Future of Waqf Institutions: between the fixed and the variable, Awqaf journal, Seventh Issue, Fourth Year, November 2004, Kuwait: Kuwait Awqaf Public Foundation, pp. 61-82

Barnes, J.R. (1989). An Introduction to Religious Foundations in the Ottoman Empire. Leiden. Review of Mr. Radwan, Journal of Ijtihad, No. 3, Year 1989, pp. 293-299.

Knew, Kenneth. (2005). Ideology and Fiqh Rhetoric. Translated by Abu Bakr Ahmed Baqader, Awqaf journal, Fifth Year, Spring 2005, Kuwait: Kuwait Awqaf Public Foundation, pp. 59-87.

Tazi, Abdel Hadi. (1995). Use of Waqf to serve foreign policy in Morocco. The Endowment in the Islamic World as a Tool of Social and Political Power, Randy Deguilhem (presented), First Edition, Damascus: The French Institute for Arab Studies. 
طارق عبدالله، حاصل على الدكتوراه في علم الاقتصاد من جامعة بارس 10، ودكتوراه في علم

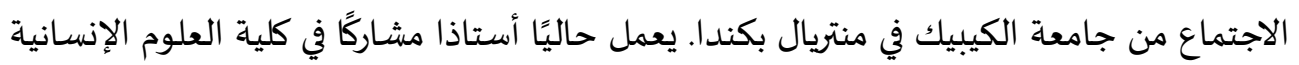
والاجتماعية بجامعة زايد بدولة الإمارات العربية المتحدة. من اهتماماته البحثية دراسة التغييرات الاجتماعية في العالمين العربي والإسلامي مع التركيز على نشأة وتطور الأنساق الاجتماعية الفرعية مثل: الوقف والمؤسسات المهنية في تفسير حركية الأفراد والجماعات، وما ينتج عنها من توجهات اقتصيادية

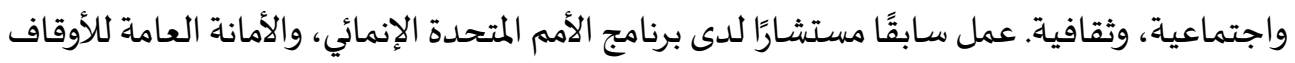

بلدولة الكويت. البريد الإلكتروني: Tarak.Abdallah@zu.ac.ae 


\title{
Toward a second wave of waqf revival in Islamic World
}

\author{
Tarak Abdallah \\ Associate Professor, Sociology, College of Humanities and Social Sciences \\ Zayed University, United Arab Emirates
}

\begin{abstract}
The last decade of the twentieth century had shown a clear intellectual effort that target reviving waqf after long decades of disinterest. Two main tendencies dominated contemporary Arabic literature relating to Awqaf; the first focuses on the definition of Waqf, its jurisprudence stipulations, and legal requirements, the second highlightes the role played by Waqf in different aspects; social, cultural and economic levels. However, these roles, as described for instance by Mohammed Mowaffaq Arnaout's paper, were achieved because of a complex social setting where Waqf was a genuine integrated part of the whole Islamic societal system. The successes of waqf roles were mainly accomplished through a specific social structure, where different sub-systems (waqf was one of them) interconnect together to allow a balanced management of the entire Muslim society. Therefore, analyzing the potentials of Waqf, its importance and role in contemporary societies, is in fact strongly correlated to the availability of specific conditions and situations where the essence of waqf could find a proper environment to bloom, so that it is able to produce its social benefits. In that context, a second intellectual revival wave is a necessity to reconsider a waqf fruitful contribution to tackle our diverse social challenges.
\end{abstract}

Keywords: Waqf, Social systems, Waqf Revival

KAUJIE Classification :E21, E22, E23 